\title{
Thickened earlobes-conductive deafness syndrome
}

INSERM

\section{Source}

INSERM. (1999). Orphanet: an online rare disease and orphan drug data base. Thickened earlobes-conductive deafness syndrome. ORPHA:2405

Thickened earlobes-conductive deafness syndrome is characterized by microtia with thickened ear lobes, micrognathia and conductive hearing loss due to congenital ossicular anomalies. It has been described in two families. The mode of inheritance is autosomal dominant. 